\title{
Novel Method of Face Feature Extraction and the Realization based on DSP
}

\author{
Liu Jinqing ${ }^{1, a^{*}}$, Liu Yin ${ }^{2, b}$ and Qunzhen Fan ${ }^{3, c}$ \\ ${ }^{1}$ Department of information, Fuzhou College of Foreign Studies and Trade, \\ Fuzhou,Fujian, China, 350202 \\ ${ }^{2}$ College of Photonic and Electronic Engineering, Fujian Normal University, \\ Fuzhou,Fujian, China,350007 \\ ${ }^{3}$ Jinshan College of Fujian Agriculture and Forestry University,Fuzhou,China,350002 \\ aemail:jqliu82088@sohu.com, bemail:liuyin941120@ sohu.com, email:309428110@qq.com
}

\begin{abstract}
Keywords: Feature extraction; Face recognition; Discrete Wavelet Transform (DWT); Principal Components Analysis (PCA); Linear Discriminant Analysis (LDA).

Abstract. Face recognition is an attractive article in pattern recognition and artifical intelligence field. From the actual requirements, the DSP hardware structure is introduced, two feature extraction algorithms are used; and then a method is proposed. the method is based on combination Discrete Wavelet Transform (DWT) with Principal Components Analysis (PCA) and Linear Discriminant Analysis (LDA), and nearest neighbor is used as a classifier. The results evaluated using the classification and recognition of ORL face database. Finally in order to verify the real-time, CCS development environment optimization program is used to achieve the algorithm in DSP platform.Experimental results demonstrated that recognition rate and recognition speed are all satisfactory improvemented.
\end{abstract}

\section{Introduction}

In recent years, biometric technologies have been widely applied in the field of public safety and financial services, and as a booming new industry. Face recognition is an important biometric identification technology and its great prospect has increasingly become a hot research topic in the current field of pattern recognition[1]。Face as the main facial features, has features of directly, friendly, convenient and informative, as the most directly means of authentication.Feature extraction[2] is an important step in face recognition, it will directly affect the classification results. Traditional face recognition systems are accomplished by large-scale or ultra LSI, the graphics workstation or microcomputer fulfill device driver and image acquisition, make image acquisition depends on the large equipment, slower, less real-time, the price is more expensive.

The Digital Signal Processor (DSP) is the core of high-performance system, stability, repeatability, can be large-scale integration and so on, not only has the programmability and real-time run much faster than general-purpose microprocessor, giving the image real-time processing has brought tremendous development[3].From the practical, TI company DM642 chip as hardware core, integrated development software CCS (Code Composer Studio) as software platform, a novel method for face feature extraction be proposed, the program can be used in real-time.

\section{Facial Feature Extraction Algorithm Theory}

DWT Algorithm. Wavelet analysis is considered to be a major breakthrough in mathematical analysis and methods, it is superior to the Fourier Transform because that it has good localization properties in time domain also frequency domain. Its essence is to use a set of multi-scale high-pass and low-pass filters for filterring, the signal's low-frequency part and high frequency part are decomposed into different frequency bands, then for further analysis and processing[4], it become a powerful tool for image analysis and signal processing. 
In order to processing image data facilitately, image processing should be data processing appropriately, just select some point in time, as well as discrete points, the continuous wavelet discretization, usually take the waveletwe two-dimensional discrete processing in the process of computer realization.After this discretization will get wavelet and corresponding wavelet transform are called discrete wavelet transform.

Two-dimensional wavelet is composed of a two-dimensional scaling function $\varphi(x, y)$ and three dimensional wavelet function, they are $\phi^{H}(x, y), \phi^{V}(x, y)$ and $\phi^{D}(x, y)$, Each is product of one-dimensional scaling function $\varphi(x)$ and wavelet function $\phi(x)$, Therefore obtain four product function are separable scale function $\varphi(x, y)=\varphi(x) \varphi(y)$ and three separable in the direction sensitive wavelet function: $\phi^{H}(x, y)=\phi(x) \varphi(y), \phi^{V}(x, y)=\varphi(x) \phi(y)$ and $\phi^{D}(x, y)=\phi(x) \phi(y)$.

These wavelet function changed along the different directions of the image gray,for example $\phi^{H}(x, y)$ changed along the column direction, such as horizontal edges; $\phi^{V}(x, y)$ changed along the line direction, such as vertical edges; $\phi^{D}(x, y)$ changed along the diagonal.

For the size of $N \times M$ image $\mathrm{f}(\mathrm{x}, \mathrm{y})$ discrete wavelet transform is

$W_{\varphi}\left(j_{0}, m, n\right)=\frac{1}{\sqrt{M N}} \sum_{x=0}^{M-1} \sum_{y=0}^{N-1} f(x, y) \varphi_{j_{0}, m, n}(x, y) \quad W_{\phi}^{i}(j, m, n)=\frac{1}{\sqrt{M N}} \sum_{x=0}^{M-1} \sum_{y=0}^{N-1} f(x, y) \phi_{j, m, n}^{i}(x, y)$

$i=\{H, V, D\}, \mathrm{j}_{0}$ is arbitrary starting scale, $W_{\varphi}\left(j_{0}, m, n\right)$ factor is defined $f(x, y)$ approximation in scale of $j_{0}, W_{\phi}^{i}(j, m, n)$ coefficient for the $j \geq j_{0}$ details of horizontal, vertical and diagonal. In general $j_{0}=0$, $N=M=2^{J}, j=0,1,2 \ldots, J-1, m, n=0,1,2, \ldots, 2^{j}-1$ 。

An images can be viewed as two-dimensional matrix, it can be supposed that the size of the image matrix is $N \times M, N=2^{n}$ ( $n$ is non-negative integer). After each Wavelet Transform, image is decomposed into four quarter-size images, they are all generated by the original map and a little Porgy Figure like the inner product,then in rows and columns direction for 2 times interval sampling. Figure 1 shows schematic diagram of the wavelet decomposition.

\begin{tabular}{|l|l|}
\hline LL1 & HL1 \\
\hline LH1 & HH1 \\
\hline
\end{tabular}

\begin{tabular}{|c|c|c|}
\hline LL2 & HL2 & \multirow{2}{*}{ HL1 } \\
\cline { 1 - 1 } LH2 & HH2 & \\
\hline LH1 & HH1 \\
\hline
\end{tabular}

Fig. 1 two-dimensional discrete wavelet transform decomposition diagram

Figure 2 shows the wavelet decomposition process of the Yale face database a human face, the low frequency component of two-dimensional decomposition is the original image's $1 / 16$. It can be seen from Figure 2, the most information of the face image is concentrated in low-frequency part, An image for n-dimensional wavelet transform[5], the low-frequency sub-band is only the original size's $1 / 2^{2 n}$. This is not only reduce image dimension, but also achieve purpose of reducing the complexity of the follow-up algorithm. This article first make face images to two-dimensional wavelet decomposition, then take the low-frequency part for subsequent feature extraction and recognition.
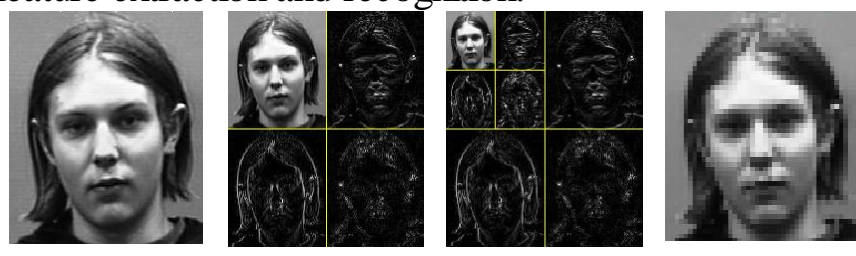

(a) Original image (b) one-wavelet decomposition (c) two-wavelet decomposition (d) low frequency components Fig. 2 face image wavelet decomposition

Eigenface Method. Eigenface method, also known as Principal Component Analysis (PCA), is a statistical method based on the overall gray, whose basic principle is use KL transform of face images[6].Assuming that the face is in a low-dimensional data space, and different expression has the separability, the face images by KL transform to obtain a new set of orthogonal basis. If eigenvalues of the KL transform matrix sorted in descending order $\lambda_{1} \geq \lambda_{2} \geq \ldots \geq \lambda_{m} \ldots \geq \lambda_{M}$, then the corresponding 
eigenvectors $W=\left[e_{1}, e_{2}, \ldots, e_{m}\right], m \leq M$. That the face image is decomposed into a number of feature vectors, these vectors are arranged out of the features of "human face" so that they are called "eigenface". And original facial image can be reconstructed from the part of the weighting coefficient of eigenface. Fig. 3 gives the experimental principal components of the ORL face database[7].Selected former main feature vector of $\mathrm{m}$ $(m<M)$ as the orthogonal basis, training samples and identified images were projected onto the new $\mathrm{m}$-dimensional space represented, get projection coefficients to be recognize faces. This time face recognition problem is converted into coordinates coefficient classification problem.
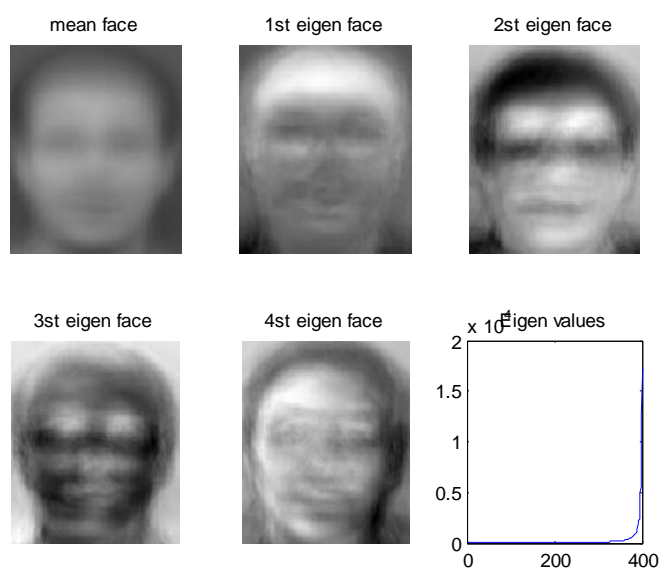

Fig. 3 Principal components of ORL face database

Linear Discriminant Analysis. Linear discriminant analysis (LDA), is an improvement method on the basis of eigenface, whose purpose to make the most discriminant ability of low-dimensional feature to extracted from the high-dimensional feature space, and these low-dimensional feature can make as much as possible to separate different types of samples, as much as possible to together the same class sample, which is to maximize the between-class distribution, to minimize class distribution. Assume that belongs to the class $c \quad \mathrm{~N}$-facial image $\left\{X_{1}, X_{2}, \ldots, X_{N}\right\}$, the between-class scatter matrix, $s_{b}=\sum_{i=1}^{c} N_{i}\left(m_{i}-m\right)\left(m_{i}-m\right)^{T}$, within-class scatter matrix $s_{w}=\sum_{i=1}^{c} \sum_{x_{k} \in X_{i}}\left(x_{k}-m_{i}\right)\left(x_{k}-m_{i}\right)^{T}, m_{i}$ is mean of $c_{i}$ class sample, $m$ is mean of all samples. LDA method, the goal is to get a projection direction $W_{\text {opt }}$, which make the ratio between class dispersion and within-class dispersion is maximum, to meet $J\left(W_{\text {opt }}\right)=\arg \max \frac{\left|W^{T} S_{b} W\right|}{\left|W^{T} S_{w} W\right|}$.

However, in practical applications, usually does not use this method for facial feature extraction directly, which is prone to small sample size problem, the number of training samples is much less than the dimension of image vector, caused the within-class scatter matrix $s_{w}$ is singular.

In order to solve this problem, improved method is combination PCA with LDA, Fisherfaces method[8], also known as FLD method. In this method, first uesd PCA method to reduce the dimensionality of the original face images, then face images projected onto a low-dimensional feature subspace, in order to ensure the sample within-class dispersion matrix non-singular, then in this feature subspace use LDA method, training a classifier for optimal discrimination.

The Steps of Algorithm Implementation . The entire recognition process is divided into two stages, namely training phase and recognition phase.

For the training phase:

(1) First, all the training sample are pretreatment, before generation of eigenface, the face image by two-dimensional discrete Wavelet Transform to extract the low-frequency sub-image instead of original image. If the original training samples is $N$, for one-Wavelet Transform, a picture is broken down into four different frequency bands subgraph can get $4 N$ sub-image. Due to the low-frequency sub-image with good stability, and also reduces the computation amount. 
(2) Calculation eigenface face database. the low frequency sub-images of each training samples for PCA transform, to construct the eigenface subspace. With such a dimension reduction subspace, any one face image is mapped to the sub-space corresponds to a point in the subspace; At the same time, any point in the subspace corresponds to an image, thus serve as the basis for face recognition.

(3) On this basis, calculate of the LDA method feature subspaceand characteristic coefficient vector library.

For the recognition phase:

(1) Determine one identify object, first for test image preprocessing, including image normalized, gray-scale transformation, binarization and so on.

(2) The processing identify face images,first for corresponding avelet transform decomposition of the layers and the training sample, and then the low-frequency part projected into the corresponding feature subspace.

(3) Last using the nearest neighbor recognition, to calculate the Euclidean Distance between the test image and all training sample, when the shortest distance description the recognized image may be someone in the library; To determine to be recognized image is someone person, required similarity $S$ to further judgment. Compare similarity $S$ with the human face similarity threshold $\theta$, if $\mathrm{S}>\theta$, determine the test image to one person in the library.

Identification method based on the above steps, algorithm flow chart is shown in Figure 4.

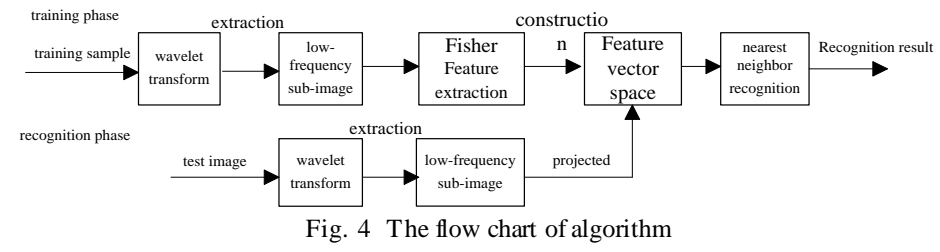

\section{System implement base on the DSP platform}

System Hardware Components. Within the DSP chip uses the Harvard architecture of program and data separately, dedicated hardware multiplier, and use pipelining.In the architecture using Very Long Instruction Word (VLIW) architecture ${ }^{[9]}$. The TMS320DM642 is TI's a powerful DSP chip in the field of multimedia processing, mainly for digital media.Its clocked at $720 \mathrm{MHz}$, with six parallel arithmetic logic unit and two parallel hardware multiplier, and a 64 External Memory Interface (EMIFA), its high-speed Digital Signal Processing capability has an irreplaceable advantage.

Based on the DM642 face recognition system block diagram shown in Figure 5. The system is core of DM642, composed of video input, video output, and memory modules.

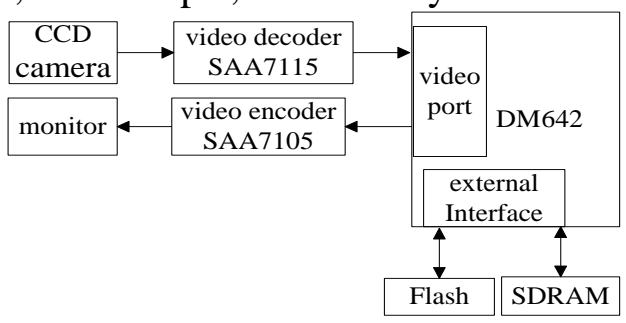

Fig. 5 System hardware flowcharts

Algorithm Optimization. In the design of software, not only to achieve the basic system functions but also consider the performance of programs, so that the entire software system can be run under the optimal and the most rapid. In order to achieve real-time face recognition, the key is how to ported the algorithm into the DSP. Very large amount of computation in the algorithm, so have to consider first algorithm is optimized.

First, effective use of Pipelining Technology, select the compiler-o2 or-o3 option,according to the procedure as much as possible to arrangements for software Pipelining ${ }^{[10]}$, in order to make full use of the software pipelining to improve operational efficiency. Followed by use of the EDMA to move data, due to 
limited resources of the DSP chip, the data is stored out the chip, useful data will be move into the chip to use EDMA. It should give full play to the role of cache memory cache,make the data in the cache be reused as much as possible. C64x compiler inline functions, improve program efficiency.

When the original design, the use of the $C$ language development, shorten the development cycle, thereby increasing the efficiency of program development. Due to the special structure of the DSP device ${ }^{[11]}$, make that the low efficiency of the $C$ compiler on the platform, compiler-generated assembly code contains a lot of redundant and therefore can not give full play to DSP's powerful computing capabilities. So, it need to optimized C code.

Use CCS profile tools to analyze $C$ code, if the low performance of $C$ code, you can use the linear assembly optimization ${ }^{[12]}$. The entire system using the $C$ language and linear assembly combination of programming. The software programming is divided into three steps: generate $C$ code phase, optimized $C$ code stage, writing linear assembly code stage. Improve system efficiency using the $C$ language, and the main achievement of image processing algorithms and system control, and assembly language to improve system performance, and the main achievement of system initialization and some optimization.

Software Design. The system software architecture is similar to system hardware, and also consists of four parts: image acquisition part of the processing section, storage section and output section. Shown in Figure 6. Feature extraction design flow, such as 7.

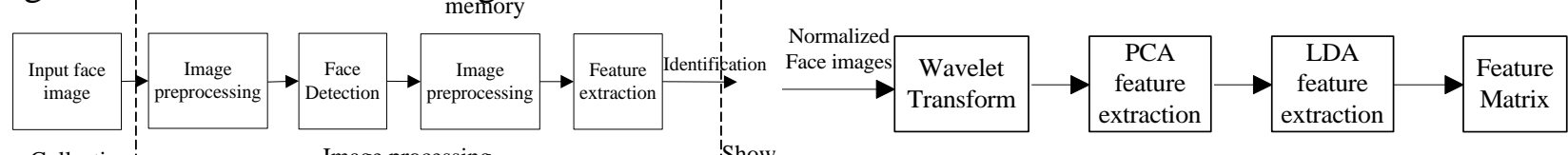

Collection

Image processing

Show

Figure 6 system software flow chart

Fig. 7 facial feature extraction flow diagram

\section{Experimental Result and Analysis}

In the experiments ORL face ${ }^{[13]}$ database is used which contains a total of 400 images, 40 individuals. Each person has 10 different images and dimension for each image is $112 \times 92$. each of the former 5 (a total of 200) images are regarded as the training sample set, and the remaining 200 images are regarded as testing samples. Using $N N$ classifier for classification ${ }^{[14]}$ to calculate the recognition rate. With the increase in the number of training, Table 1 is four ways of PCA, FLD, 2DWT+PCA and 2DWT+FLD changed rate. Table 2 isthe training and classification time of different method.

\begin{tabular}{|c|l|l|l|l|l|c|}
\hline \multicolumn{1}{c}{ Table 1 various methods of face recognition rate of ORL face database } \\
\hline $\begin{array}{c}\text { Training } \\
\text { number }\end{array}$ & 5 & 6 & 7 & 8 & 9 & $\begin{array}{c}\text { Average } \\
\text { recognition rate }\end{array}$ \\
\hline PCA & 0.81 & 0.84 & 0.85 & 0.87 & 0.88 & 0.844 \\
\hline FLD & 0.84 & 0.88 & 0.90 & 0.93 & 0.95 & 0.900 \\
\hline 2DWT+PCA & 0.8438 & 0.8833 & 0.90 & 0.95 & 0.9889 & 0.9132 \\
\hline 2DWT+FLD & 0.9063 & 0.9333 & 0.9625 & 0.9750 & 1.00 & 0.9554 \\
\hline
\end{tabular}

\begin{tabular}{|l|c|c|}
\hline \multicolumn{2}{|c|}{ Table 2 compared training time with recognition time } \\
\hline method & training time & recognition time \\
\hline PCA & $234.2 \mathrm{~s}$ & $3.12 \mathrm{~s}$ \\
\hline FLD & $212.3 \mathrm{~s}$ & $2.49 \mathrm{~s}$ \\
\hline 2DWT+PCA & $74.84 \mathrm{~s}$ & $2.02 \mathrm{~s}$ \\
\hline 2DWT+FLD & $60.47 \mathrm{~s}$ & $1.38 \mathrm{~s}$ \\
\hline
\end{tabular}

It can be seen from Table 1 experimental results: the FLD linear discriminant has high recognition rate than eigenface method. In this paper, combined the Wavelet Transform with FLD method, which greatly improve the recognition rate. Can be seen from Table 2, using combined method not only improve the recognition rate, at the same time improve the recognition speed.

In this paper, the realization of a complete system for facial feature extraction, and comparative analysis the results. In the experiments carried out in different ways, and experiments show this article wavelet analysis and Fisherfaces combine algorithm is feasible. 


\section{Acknowledgment}

The authors gratefully acknowledge the supports from The National Natural Science Foundation of China(61179011), Natural Science Foundation of Fujian Province(2010J01327), Fujian Provincial Key Laboratory of Photonics Technology and Fujian Education Department Technology Foundation (JB07040). The authors also acknowledge the editor and colleagues who provided technical supports.

\section{References}

[1] Samal, A Automatic recognition and analysis far humaa faces andfacial expressions A survey. Pattern Recognition, 1992, 25:65 77 .

[2] X. D. Liu,Z. Q. Chen, Journal of Computer Research and Development,2004,41(7):1074-1080

[3] DING W,YAN W Q,QI D X, Digital image scrambling[J].Progress in Natural Science, $2001,11(6): 454-460$.

[4] K. Q. Wang,J. K. Zhu,X. B. Bai, Techniques of Automation and Application,2009, 28(4):65-68. (In Chinese)

[5] K. Z. Yin, W. G.Gong, W. H. Li and Y. X. Liang, Chinese Journal of Scientific Instrument, Vol. 26 (2005) No.8, pp.412-415 (In Chinese).

[6] Q. C. Yu, Face Recognition Algorithm Research base on K-L transform. (Master's degree paper of Shenyang University of Technology, China 2007). (In Chinese)

[7] Yuen P C,Lai J H, Face Recognition using independent component analysis[J].Patten Recognition,2002(35):1247-1257.

[8] Yu Hua, Yang Jie, A direct LDA algorithm for high-dimensional-data with application to face recognition. Pattern Recognition, 2001, 34(10): 2067 -2070.

[9]TMS320DM642Video/ImagingFixed2Point Digital Signal Processor Data Manual[Z]. Texas Instruments Incorporated,2003.

[10]TMS320C6000 Optimizing Compiler Userps Guide[Z].Texas Instruments Incorporated, 2002

[11] H. R. Liu,G. Wang, Modern Electronic Technique , 2006(12): 92-95.(In Chinese).

[12]J. B. Hu, H. J. Chen, Modern Electronic Technique,2002(12):82-84.(In Chinese).

[13] Face Recognition Homepage, http://www.face-rec.org/.

[14] W.Zheng, L.Zhao, C.Zou, An efficient algorithm to solve the small sample size problem for LDA[J]. Pattern Recognition, 2004:1077-1079. 